\title{
HÁBITOS DE ESTUDIO, MOTIVACIÓN Y ESTRÉS ESTUDIANTIL EN AMBIENTES VIRTUALES DEAPRENDIZAJE
}

\author{
STUDY HABITS, MOTIVATIONAND \\ STUDENT STRESS IN VIRTUALLEARNING \\ ENVIRONMENTS
}

\section{Dra. Heidi Angélica Salinas Padilla ${ }^{1}$}

\section{Dr. Juan José Díaz Perera²}

\section{Dra. Cynthia Daniela Alvarez Amezcua ${ }^{3}$}

\section{Mtro. Mario Saucedo Fernández ${ }^{4}$}

Universidad Autónoma Del Carmen, México

\section{$1 \quad$ hsalinas@pampano.unacar.mx} https://orcid.org/0000-0002-2260-3609 Universidad Autónoma Del Carmen, México

Doctorado en Educación, con terminación en Educación a Distancia y Diseño Instruccional. Profesor de Tiempo Completo Asociado C en la Facultad de Ciencias Educativas de la Universidad Autónoma del Carmen. En nivel posgrado es profesor del núcleo base de la Maestría en Innovación y Prácticas Educativas del padrón del PNPC de CONACYT: Diseño Curricular, Modelos y Teorías del Aprendizaje, Evaluación Tecnológica en procesos educativos. Miembro del Cuerpo Académico Consolidado de Matemática Educativa en el cual ha desarrollado diversos proyectos de investigación con financiamiento, así como múltiples publicaciones nacionales e internacionales. Cuenta con el perfil deseable de Prodep $2 \quad$ jidiaz@pampano.unacar.mx

https://orcid.org/0000-0003-2098-8020 Universidad Autónoma Del Carmen, México. Doctor en Tecnología Educativa, Profesor de Tiempo Completo Asociado C en la Facultad de Ciencias Educativas de la Universidad Autónoma del Carmen. Cuenta con Perfil Prodep. En nivel posgrado es profesor del núcleo base de la Maestría en Innovación y Prácticas Educativas del padrón del PNPC de CONACYT: Líder del Cuerpo Académico de Matemática Educativa. Tiene producción científico Académica en las Líneas de Investigación y aplicación del conocimiento: • Didáctica de las Matemática • Tecnología Educativa. Actualemente es Miembro del Sistema Nacional de Investigadores del CONACYT $3 \quad$ cynthia.alvarezam@uanl.edu.mx https://orcid.org/0000-0003-4617-9504 Universidad Autónoma Del Carmen, México Doctorado en Educación, con terminación en Educación a Distancia y Diseño Instruccional, Profesor de Tiempo Completo de la Universidad Autónoma de Nuevo León, coordinadora de posgrados de la Facultad de Ciencias de la Comunicación.

$4 \quad$ msaucedo@pampano.unacar.mx

https://orcid.org/0000-0002-7970-7353 Universidad Autónoma Del Carmen, México. Maestro en Gestión e Innovación Educativa, Candidato a Doctor en Tecnología Educativa, Profesor de Tiempo Completo Asociado C en la Facultad de Ciencias Educativas de la Universidad Autónoma del Carmen. Cuenta con Perfil Prodep. Miembro del Cuerpo Académico de Matemática Educativa. Tiene producción científico Académica en las Líneas de Investigación y aplicación del conocimiento: • Didáctica de las Matemática $\cdot$ Tecnología Educativa 


\section{RESUMEN}

El aislamiento social, la ansiedad y depresión son las principales dificultades o preocupaciones experimentadas por los estudiantes durante la pandemia COVID-19, por lo cual mostraron dificultad para adaptarse al uso de recursos tecnológicos. El objetivo principal del estudio fue describir cuál es la percepción de los estudiantes sobre su rol como estudiante virtual y cómo este nuevo contexto educativo influye en sus emociones y sentimientos, dentro de su proceso formativo. Metodología: se presenta exclusivamente el piloteo del instrumento que permita obtener información que explique la situación de los universitarios en relación con la modalidad virtual y en específico sobre su rol, hábitos de estudio y condiciones de estrés La muestra utilizada para el piloteo del instrumento, con participantes, 318 estudiantes, entre los 18 y 25 años, y los cuales están inscritos en nivel de licenciatura y con estrato socioeconómico heterogéneo. El instrumento que se aplicó fue: una encuesta tipo Liket autoadministrado, los participantes se invitaron por medio que las unidades académicas que están inscritos, Resultados: Como resultado sobresalientes, se observa que el $80.7 \%$ de los estudiantes y encuestados están de acuerdo que los hábitos de estudio son esenciales para tener un buen desempeño académico, sin embargo sólo $65.9 \%$ señala que a menudo utiliza técnicas de estudio que me faciliten mi aprendizaje como los mapas mentales o conceptuales, esquemas, fichas bibliográficas entre otros. $Y$ el $73.8 \%$ consideran que la forma en que los profesores hacen llegar los nuevos conocimientos influyen en su motivación escolar, y este aspecto de apoyo por parte del facilitador, $64.7 \%$ de los estudiantes están de acuerdo que el apoyo que dan los profesores es un aspecto importante para tu motivación. Conclusión: Hasta este momento el instrumento en esta fase de prueba genera información de relevancia para conocer la percepción de los estudiantes universitarios en relación con su rol como estudiante en modalidad virtual, sus hábitos de estudio y su situación emocional.

\section{PALABRAS CLAVE}

Validación de Instrumento, Virtualidad, Hábitos Estudio, Emociones

\section{ABSTRACT}

Social isolation, anxiety and depression are the main difficulties or concerns experienced by students during the COVID-19 pandemic, thus improving the ability to adapt to the use of technological resources. The main objective of the study was to describe what is the perception of students about their role as virtual student and how this new educational context influences their emotions and feelings, within their training process. Methodology: exclusively the pilot of the instrument is presented that allows obtaining information that explains the situation of university students in relation to the virtual modality and specifically about their role, study habits and stress conditions The sample used for the pilot of the instrument, with participants, 318 students, between the ages of 18 and 25, and who are enrolled at the undergraduate level and with a heterogeneous socioeconomic stratum. The instrument that was applied was: a selfadministered Liket type survey, the participants were invited through the academic units that are enrolled, Results: As an outstanding result, it is observed that $80.7 \%$ of the students and respondents agree that the habits Study are essential to have a good academic performance, however only $65.9 \%$ indicate that they often use study techniques that facilitate my learning such as mental or conceptual maps, diagrams, bibliographic records, among others. And $73.8 \%$ consider that the way in which teachers convey new knowledge influences their school motivation, and this aspect of support from the facilitator, $64.7 \%$ of students agree that the 
support given by teachers is an important aspect for your motivation. Conclusion: Until now, the instrument in this test phase generates relevant information to know the perception of university students in relation to their role as a student in virtual mode, their study habits, and their emotional situation.

\section{KEYWORDS}

Instrument Validation, Virtuality, Study Habits, Emotions

\section{INSTRUCCIÓN (ANTECEDENTES)}

Debido a la llegada del COVID-19 el 28 de febrero del 2020, la Secretaría de Salud de México tomó acciones inmediatas con el objetivo de reducir la velocidad de su propagación en la población. A partir de marzo de 2020 las Instituciones de Educación Superior (IES), transitaron toda su oferta educativa de la modalidad presencial a la virtual para mantener la continuidad pedagógica de la educación formal a través de plataformas digitales.

En este contexto, el sistema educativo se ubica ante un proceso progresivo y contextualizado de virtualización de los diferentes procesos sustantivos de la universidad (la formación, la investigación y la extensión); lo que conduce a transformar las disciplinas, el papel del docente, del estudiante y de la propia universidad, es por esto que el uso de las tecnologías de la información y comunicación (TIC'S) como herramienta para promover la enseñanza y el aprendizaje a distancia (e-learning) ha mostrado ser eficaz y de mucha ayuda. Ya que permite incrementar la calidad de los procesos pedagógicos a distancia y la adaptación del contenido instruccional a las necesidades educativas de la comunidad académica en periodos de gran demanda (Area \& Adell, 2009; Saini, \& Mitteer, 2020).
Sin embargo, bajo la coyuntura del COVID-19, las demandas académicas, tecnológicas y psicológicas (producto de la implementación abrupta de modelos de aprendizaje a distancias y dificultades en el desarrollo de políticas de implementación y capacitación eficientes) han aportado a que el estudiante universitario experimente dificultades y barreras, independientemente de tener una actitud positiva hacia el uso de Tecnologías de la información en el proceso de enseñanza y aprendizaje (Sanz, Sainz y Capilla, 2020).

Hay que tomar en cuenta el cambio abrupto en la modalidad de enseñanza y aprendizaje generado por la propagación de CO-VID-19 representa un impacto inmediato en ciertas dificultades en los estudiantes en el sistema de educación superior (Regmi \& Jones, 2020). Otros estudios realizados recientemente sugieren que el aislamiento social, la ansiedad y depresión relacionada al COVID-19, la dificultad para adaptarse a las nuevas tecnologías, las expectativas de mantener un horario y escenario académico regular, aspectos económicos y la accesibilidad a recursos tecnológicos son las principales dificultades o preocupaciones experimentadas por los estudiantes durante la pandemia (Instituto Internacional para la Educación Superior en América Latina y el Caribe, 2020).

Tal como explica Salazar (2020), esto implica reflexionar sobre cómo transformar aspectos como la estructura organizativa curricular, los tiempos, las asignaturas, los espacios, las clases, las estrategias pedagógicas (diseño de materiales virtuales), la incorporación del trabajo remoto, sincrónico y colaborativo en los procesos de enseñanza-aprendizaje virtuales; especialmente en la construcción de recursos educativos, en la creación de contenidos, en la apariencia visual del aula virtual y la falta de capacitación profesional de los profesores hacia el manejo adecuado de las TIC'S. 
Por lo tanto, uno de los primeros desafíos con lo que se encuentran los directivos y los docentes como agentes mediadores del proceso educativo, es que deben saber cómo integrar a su quehacer las estrategias metodológicas que van a utilizar para asegurar que los procesos de enseñanza aprendizaje no pierdan su esencia y calidad en este proceso de virtualización.

Una de las mayores dificultades en la configuración del espacio del aula virtual son el uso de la videoconferencia como "espejo del aula presencial", sobre todo en ciertos profesores que estaban acostumbrados a clases expositivas teóricas con escaso tiempo de interacción con los alumnos, ya que esta se les ha complicado muchísimo y en algunos casos se ha vuelto una barrera para pasar sus conocimientos a los estudiantes, a nivel institucional, al igual los hábitos de estudio en los alumnos son algo fundamental en esta nueva modalidad, ya que no todos tienen buenos hábitos ni saben cómo emplearlos de manera eficiente.

Al mencionar estas problemáticas curriculares al momento de estar impartiendo una clase, es prioritario tomar en cuenta a los estudiantes que tienen problemas un poco mayores que estos, hay discentes que en esta pandemia no tienen las mismas posibilidades económicamente, sufren aún mucho más las consecuencias de la llamada "brecha educativa", la cual se identifica como la diferencia que existe entre la educación que reciben aquellos jóvenes con menos recursos y con situaciones familiares complicadas, y aquellos que provienen de entornos más estables y más adinerados.

La "cuarentena o el gran confinamiento" y la globalidad de esta enfermedad, sumada a las condiciones de interconectividad por medio de las redes sociales y los diversos medios tecnológicos han puesto en evidencia las desigualdades socioeconómicas y los rasgos de incertidumbre del mundo en que vivimos.
Boaventura de Sousa Santos (2020) señala que se trata de una crisis grave y aguda, donde "la cuarentena no sólo hace más visibles, sino que también refuerza la injusticia, la discriminación, la exclusión social y el sufrimiento inmerecido que provocan".

Según el PNUD (2019), vivimos en la región con mayor desigualdad en los ingresos de todo el mundo. La disponibilidad de las TIC's y, en algunos casos, hasta de los medios tradicionales como televisión y radio, reproduce la desigualdad. En nuestro país encontramos estas inequidades y mucho retraso en las inversiones físicas en las escuelas, la conectividad de banda ancha, el equipamiento, el software y la formación de los trabajadores de la educación en esta materia.

Las posibilidades de trabajo sincrónico entre maestros y estudiantes, el número y tipo de recursos tecnológicos utilizados, o las condiciones para dar marcha a la educación digital hacen evidente las diferencias entre modalidades y tipos educativos, escuelas privadas y escuelas públicas, entre el medio rural y el urbano, entre zonas industrializadas y de mayoría indígena, etcétera. Estas diferencias potencian la exclusión y el rezago educativos, obstaculizan el ejercicio ciudadano de la libertad y de la democracia, y mantienen el círculo de la pobreza y la inequidad (Chehaibar, L. M. 2020).

Considerando que la educación es un derecho humano fundamental, esencial para ejercer los demás derechos de los que son titulares las mujeres y los hombres de este planeta. Es un bien social y público, de amplio valor en la sociedad. Y la educación, en todos sus niveles y tipos educativos, se ha visto paralizada, encarada y retada en el contexto de la pandemia de covid-19 que afecta al mundo. Otro de los puntos fundamentales, es el punto de conexión humanizado entre docente-alumno, en este sentido, Maldonado et al., (2020) explican que es necesario interactuar con los discentes, 
comprendiendo la situación por la que estos atraviesan, tomando asimismo en consideración la diversidad de sus contextos y posibilidades.

En México, a la fecha, no se cuenta con resultados de investigaciones científicas sobre el impacto psicológico del confinamiento durante la pandemia en la población de los estudiantes universitarios. Por tal motivo, el autor Morelos (2020) realizó una investigación que consistió en evaluar los niveles de ansiedad, depresión, estrés y la percepción del estado de salud en estudiantes universitarios mexicanos durante 7 semanas de contingencia por la pandemia de Covid-19.

En el cual participaron 644 discentes (276 mujeres y 368 hombres) con edad promedio de 21.95 años $(\sigma=3.56)$, en un rango de 1843 años. El lugar de residencia referido por los participantes durante el confinamiento por la pandemia de Covid-19 se distribuyó de la siguiente manera: $4.4 \%$ en la Ciudad de México y Estado de México; $9.3 \%$ en el Estado de Guerrero; $57.5 \%$ en Morelos; $6.1 \%$ en Nayarit; $9.5 \%$ en Nuevo León y $13.4 \%$ en Puebla. Con respecto al número de personas que conviven en el mismo hogar con los estudiantes durante el confinamiento, se observó que el $3.9 \%$ vive solo, $8.7 \%$ vive con una persona, $16.9 \%$ con dos personas, $30.6 \%$ con 3 personas y $39.9 \%$ con más de 3 personas.

Los datos reflejados de este trabajo mostraron que, durante la fase de confinamiento, prácticamente la cuarta parte de la población en estudio, independientemente de las variables sociodemográficas, presentó dolor de cabeza, sensación de falta de control y poca satisfacción con las formas de realizar sus actividades. Aproximadamente un tercio de la población refirió sentirse agobiado, con nerviosismo, mal humor, menos activos, invirtiendo más tiempo de lo habitual al realizar las tareas cotidianas y con sentimientos de inutilidad. La incapacidad para disfrutar las actividades normales de cada día fue el síntoma que mostró mayor prevalencia con $42 \%$.

Con relación a la variable ansiedad (moderada a severa), se observó que los varones presentaron puntuaciones más altas que las mujeres. Sin embargo, las mujeres reflejaron más altos puntajes (moderados a severos) en síntomas psicosomáticos, insomnio, disfunción social, depresión y estrés. Los mayores niveles de ansiedad, estrés, depresión, síntomas psicosomáticos, dificultades para dormir y disfunción social en la actividad diaria se presentaron en los grupos más jóvenes (1825 años).

A través de este estudio, surge la necesidad de implementar estrategias preventivas, de control y disminución de los efectos provocados por el confinamiento debido a la pandemia de Covid-19 con el objetivo de promover el bienestar y la salud mental de los estudiantes universitarios (Morelos 2020), por lo cual es sumamente importante que los profesores tomen en cuenta los sentimientos y emociones que presentan sus alumnos durante este periodo educativo.

Finalmente, la propuesta que se presenta en este documento pretende compartir los resultados de la primera aplicación de un instrumento que busca obtener información sobre cuál es la percepción de los estudiantes sobre su rol como estudiante virtual y cómo este nuevo contexto educativo influye en sus emociones y sentimientos.

Para ello se plantean los cuestionamientos ¿Cuál es la perspectiva de los estudiantes universitarios acerca de su rol en modalidad virtual?, ¿los universitarios tienen hábitos de estudios que les ayuden a continuar con su formación? ¿Qué condiciones de estrés genera la modalidad virtual y el confinamiento en los universitarios? Esta primera corrida del 
instrumento busca mejorar el instrumento de recolección de datos, que permita generar algunas alternativas de mejora para identificar las condiciones de estrés en los estudiantes universitarios, como consecuencia de su desempeño académico en la modalidad virtual a causa de pandemia.

La aplicación del instrumento se llevó a cabo en una Universidad del Norte de la República Mexicana, en una dependencia de formación de las Ciencias Sociales y las Humanidades; durante el ciclo escolar septiembre-diciembre 2020, en modalidad en línea. El alcance se supedita exclusivamente al piloteo de un instrumento que permita obtener información que explique la situación de los universitarios con relación a la modalidad virtual y en específico sobre su rol, hábitos de estudio y condiciones de estrés ante este nuevo contexto educativo; esta prueba piloto, tendrá un alcance explicativo acerca del comportamiento de los ítems del instrumento.

\section{JUSTIFICACIÓN}

La importancia de realizar esta investigación es contar con un instrumento de medición que brinde certeza sobre la forma en la que impactó a los estudiantes universitarios la modalidad 2020 por la pandemia sanitaria del COVID-19, ya que estaban acostumbrados a tener clases de manera presencial lo cual conlleva responsabilidades diferentes a un estudiante en modalidad virtual. En consecuencia, se requiere analizar que tanto ellos conocen acerca del rol que juegan al ser estudiantes a distancia y cuál es la situación emocional por la que están pasando y que tanto les afecta en su formación profesional.

Cabe destacar que la modalidad virtual es algo que se ha ofertado ya hace unos años en la educación a través de diversos modelos instruccionales, sin embargo, hay que tomar en cuenta que nadie estaba preparado para tomar clases por medio de la vía online, y que a algunos profesores y alumnos se les complica esta nueva modalidad. De igual forma, este escenario da lugar a grandes preocupaciones dada la posibilidad de que se genere un periodo de vulnerabilidad que promueva el abandono académico a gran escala (UNESCO, 2020). Dicho planteamiento promueve de gran manera el desarrollo de este el presente proyecto de investigación, buscando así identificar rutas y elementos que faciliten la implantación eficiente de modelos de aprendizaje en momentos de gran demanda.

Las variables que se interpretaran en este proyecto son:

\ Hábito de estudio: Los hábitos de estudio son los métodos y estrategias que utiliza el estudiante para comprender los diversos temas dentro de las unidades de aprendizaje, su aptitud para evitar distracciones, su atención al material específico y los esfuerzos que realiza a lo largo de todo el proceso (Cartagena, 2008).

Q Emociones: es el conjunto de reacciones orgánicas que experimenta una persona como respuesta a ciertos estímulos externos que le permiten adaptarse a una situación con respecto a una persona, objeto o lugar, estas pueden ser de tipo psicológico, conductual o fisiológico.

\section{MARCO TEÓRICO-CONCEPTUAL}

Conocer cuál es el rol del estudiante en modalidad virtual es un elemento importante en los procesos de formación que se viven actualmente, ya que tanto el profesor como el alumno juegan roles dentro de la proceso de enseñanza/aprendizaje y es importante tener en claro cuál es el papel del estudiante en un entorno virtual, por lo tanto el cuestionamiento es ¿Cuáles serían los elementos que debe expresar un estudiante virtual? 
El rol del estudiante en los ambientes educativos mediados por las TIC'S, Escudero (1992) señala que la definición de los criterios, los valores y los intereses al adoptar estas debe obedecer a una planificación que permita desarrollar los procesos pedagógicos, en los que el discente desempeña un rol central representado en el conjunto de comportamientos y normas que este debe asumir como actor del proceso educativo.

Las características del rol del estudiante virtual que le permiten la generación del conocimiento están directamente relacionadas con:

$\otimes \quad$ La capacidad de autogestión: es la dedicación permanente en las tareas propuestas, fundamentada en la definición de objetivos, su ejecución, seguimiento, acompañados por la decisión, la motivación y la perseverancia, lo conducen hacia el logro de sus metas.

$\otimes \quad$ La autodisciplina: tiene la capacidad para distribuir su tiempo, permite libertad y flexibilidad para el aprovechamiento del aprendizaje mediado por las TIC's, conduciendo a la generación de movimientos de los sujetos hacia el logro de sus propias metas.

\} \quad \text { El autoaprendizaje: la capacidad que } desarrolla un individuo para aprender de manera autónoma activa y participativa, adquiriendo conocimientos y habilidades, fomentando así sus propios valores, lo que da como resultado la autoformación del sujeto (Rugeles, Mora \& Metaute 2015). Es así como el autoaprendizaje le facilita al estudiante virtual el desarrollo de la capacidad de exigirse a sí mismo, pues lo involucra en la toma de decisiones.

\} \quad \text { El análisis crítico y reflexivo: es la } habilidad para razonar, analizar y argumentar hechos o acciones que faciliten el desarrollo integral del estudiante y la generación de conocimiento. $\nabla \quad$ El trabajo colaborativo: Pretende romper el aislamiento entre los diferentes actores permitiendo ver las cualidades individuales que son compartidas entre los discentes y facilitadores a través de herramientas como el foro, el correo electrónico, las salas de conversación y los Objetos Virtuales de Aprendizaje, (OVA), (Rugeles, et. al, 2013).

\ Ética profesional: Martínez (2010) lo define como área del conocimiento humano que permite un juicio sobre el alcance de las acciones y comportamientos individuales o colectivos relacionados con los aportes constructivos o destructivos para sí.

Por lo tanto, esto permite que su proceso educativo sea más humanizante, como un sujeto que piensa actúa, crea y construye saberes personales y sociales.

El COVID-19 vino a afectar el contexto educativo de una forma radical, ya que esta pandemia a nivel global ha dado lugar al cierre masivo de las actividades presenciales de instituciones educativas en más de 190 países con el fin de evitar la propagación del virus y mitigar su impacto. Esta situación ha resultado difícil tanto para los estudiantes como para los docentes, que tienen que enfrentarse a los problemas emocionales, físicos y económicos provocados por la enfermedad, que al mismo tiempo cumplen con la parte que les corresponde para contribuir a frenar la propagación del virus.

Por su parte, la UNESCO ha identificado grandes brechas en los resultados educativos, que se relacionan con una desigual distribución de los docentes, en particular, en países y regiones con menores ingresos y de zonas rurales, las que suelen concentrar además a población indígena y migrante.

Desde esta perspectiva, la escuela comprendida como la institucionalización del proceso 
formativo socialmente válido, impartida ya sea por el Estado o por la administración privada, ha tenido que adecuarse al contexto de la pandemia por Covid-19. Según la Organización de las Naciones Unidas para la Educación, la Ciencia y la Cultura (UNESCO, 2020), alrededor de $70 \%$ de la población estudiantil del mundo se está viendo afectada; mientras que en México (preescolar, primaria, secundaria, media superior y superior) un total de 37589960 estudiantes se han visto afectados. Estos números se agravan cuando se promueve la idea de que el curso escolar debe continuar; según el World Economic Forum (WEF, 2020, s. p.).

Hay que tomar en cuenta que, con respecto a los hogares con conectividad a Internet se alcanza 56.4\% (INEGI, 2015-2018). En contexto del Covid-19 en educación superior, los márgenes de tiempo para abdicar son diferentes, las necesidades educativas con respecto a los conocimientos son especializados, sujetos a exigencias sociales y demandas económicas. América Latina se enfrenta a desafíos en la formación de los docentes en materia de TIC. Por ejemplo, en Brasil en 2018, sólo el $20 \%$ de los docentes participaron en un curso de educación continua para el uso de computadoras e Internet para la enseñanza.

La universidad como bien se sabe debe responder a los requerimientos sociales y al mercado del conocimiento, es decir, buscar ser pertinente, por ello, es compresible que instituciones tanto internacionales como nacionales se preocupen por la proyección de lo que la universidad produce o puede contribuir a la sociedad en este contexto de crisis sanitaria; sin embargo, el coste de esta movilidad de recursos y pronunciamientos de las universidades van en detrimento de la consideración de cómo se da el proceso de formación universitaria; esto es, la visión desde dentro del proceso formativo, puesto que al descuidarse la didáctica, lo que se esté produciendo en la educación superior puede llegar a no cumplir con las expectativas sociales y económicas. A partir de esto se debe tener presente que hay una matrícula de 3,943 , 544 millones de estudiantes a nivel superior en México (SEP, 2019).

Los actores directos del proceso formativo en educación superior se ven rebasados en diferentes ámbitos, en específico, en dos: las condiciones estructurales, como son las tecnológicas, cantidad de dispositivos con los que cuenta, el espacio geográfico y la conectividad a Internet; por otra parte, se encuentran los pertenecientes a los que están en sus posibilidades como personas: los socioemocionales, las nuevas formas de aprender, las competencias digitales, la comunicación y la organización eficaz.

Tanto estudiantes como docentes manifiestan requerir apoyo y ver como obstáculo la nueva modalidad emergente de tomar clases de manera virtual. Esto quiere decir que la edad o generación no es un determinante para saber más o menos sobre las tecnologías; esto nos muestra que la educación actual está en crisis debido a que existe un desajuste entre los avances tecnológicos, la currícula, las metodologías y las necesidades de los estudiantes.

Son diversos los factores que pueden influir en el rendimiento escolar, pero cuando un alumno que está suficientemente capacitado o dotado fracasa y no hay otra causa aparente, este fracaso se relaciona estrechamente con unos hábitos de estudio incorrectos llega un punto en el cual es necesario un método, un sistema de trabajo que le permita al discente cursar con éxito los estudios.

El autor Cartagena (2008), los hábitos de estudio son los métodos y estrategias que utiliza el estudiante para comprender diversos temas dentro de las unidades de aprendizaje, su 
aptitud para evitar distracciones, su atención al material específico y los esfuerzos que realiza a lo largo de todo el proceso. En base a esto se puede decir que hay hábitos de estudio buenos y malos, o más bien correctos e incorrectos.

Algunos de los hábitos de estudio muy comunes en los estudiantes son la memorización, tratar de incorporar el contenido a aprender a último momento, tratar de aprender en un lugar con poca luz, desordenado o ruidoso, ser desprolijo y estudiar cansado o con hambre, estos son considerados como malos hábitos, por lo cual se deben tratar de eliminarlos de nuestro quehacer continuo.

Los hábitos de estudio que promueven un buen desarrollo profesional y académico que se pueden aplicar son:

- Cumplir horarios (organización de tiempo)

- $\quad$ Establecer una metodología de trabajo.

- Estudiar en un lugar silencioso y cómodo.

- Utilizar técnicas para aprender significativamente como puedenser: elsubrayado de un texto, cuadros sinópticos, comparativos o mapas conceptuales, repasar; exponer en voz alta lo aprendido, realizar apuntes; releer si no se entiende; ayudarse con el diccionario para realizar la lectura comprensiva.

- Tomar descansos durante las horas de estudio también es sumamente importante.

Estos se van formando en forma progresiva, con la ayuda del maestro y la familia como guías, contar con buenos hábitos de estudio ayuda a promover el desarrollo cognitivo, así como la capacidad de aprender con menor dificultad nuevos contenidos y mejorar el rendimiento académico posibilitará que el alumno ya no cuente con la guía permanente de sus docentes y pueda transformarse en alguien capaz de estudiar por sí solo, que es lo que se denomina estudiante con autonomía, además de que esos contenidos se acumulan en su memoria de largo plazo.

Lo anterior, va de la mano con las emociones que pueden presentar los estudiantes que se encontraban en modalidad presencial antes de la pandemia, y como ya se ha mencionado ha sido un cambio drástico el proceso y la adaptación de este nuevo contexto educativo para los discentes de todos los niveles académicos, ya que si los alumnos no tienen buenos hábitos de estudio generará en ellos un estrés más a esta nueva modalidad.

Se debe recordar que las emociones emergen como respuesta ante una situación que se esté viviendo, poseen una función adaptativa con respecto al entorno. En este sentido, el impacto emocional de la cuarentena en niños, adolescentes y jóvenes adultos está asociado con diferentes emociones como el miedo, el aburrimiento, irritabilidad, inquietud motora, cambios de humor repentinos e incluso tristeza profunda por falta de contacto con sus familiares, por la situación económica, la diferencia metodológica a la que se tiene en el nuevo contexto educativo.

El cierre de los centros educativos, la necesidad del distanciamiento físico, la pérdida de seres queridos, del trabajo y la privación de los métodos de aprendizaje convencionales han generado estrés, presión y ansiedad, especialmente entre los docentes, el alumnado y sus familias, asegura la (UNESCO, 2020).

Los trastornos que se generan ante este contexto privan a los alumnos de una estabilidad emocional requerida para atender su formación profesional con plenitud, ante esta problemática mundial. Las condiciones que acompañan 
a esta pandemia incluyen distintas fuentes de estrés para los estudiantes. Algunos de los estudios sobre situaciones de estrés y emergencias permiten resumir las principales variables implicadas en el impacto psicológico como las siguientes: el miedo a la infección por virus y enfermedades, la manifestación de sentimientos de frustración y aburrimiento, no poder cubrir las necesidades básicas y no disponer de información y pautas de actuación claras (Brooks, 2020).

\section{METODOLOGÍA}

La presente investigación se basó en el enfoque cuantitativo, de carácter exploratorio; la población a la que se le aplicó el instrumento de recolección de datos en su prueba piloto se compuso por los estudiantes del ciclo escolar septiembre - diciembre 2020 de una universidad al norte de la República Mexicana, mientras que la muestra fueron 318 discentes en el plan de estudios de comunicación, el cual cuenta con ocho semestres y se piloteo con los activos del ciclo escolar mencionado. La técnica de recolección de datos utilizada fue una encuesta tipo Likert vía online, el cual es un método de medición utilizado por los investigadores con el objetivo de evaluar la opinión y actitudes de las personas, es una escala de calificación que se utiliza para cuestionar a una persona sobre su nivel de acuerdo o desacuerdo con una declaración, es ideal para medir reacciones, actitudes y comportamientos de una persona.

El diseño del instrumento contempla tres áreas temáticas: hábitos de estudio, rol del estudiante en modalidad virtual y por último, situación emocional y de estrés en esta modalidad en línea, cada tema está integrado por 10 preguntas haciendo un total de 30 reactivos con una opción de cinco respuestas: 1 . Muy de acuerdo, 2. De acuerdo, 3. Ni en acuerdo ni en desacuerdo, 4. En desacuerdo y 5. Nada de acuerdo y para finalizar la encuesta una pregunta abierta para enriquecer un poco más la investigación, finalmente se destaca que las preguntas que integran el instrumento se plantearon a partir de la revisión de la literatura.

\section{RESULTADOS Y ANÁLISIS DE DATOS}

Como resultado de esta primera prueba piloto del instrumento aplicado, se presentan a continuación la información obtenida sobre la primer variable: hábitos de estudio, para $\begin{array}{lll}4 & 0 & 1\end{array}$ lo cual se retoma al autor Cartagena (2008), quien plantea como hábitos de estudio a los métodos y estrategias que mediante su uso - implementación por parte del estudiante le facilita comprender los contenidos, le permiten desarrollar concentración y optimizan su esfuerzo para aprender. Los resultados obtenidos en el marco de esta categoría son los siguientes: 
Tabla 1. Hábitos de estudio

\begin{tabular}{|l|l|l|l|l|l|}
\hline $\begin{array}{l}\text { En relación a tus hábitos de estudio, } \\
\text { ¿en qué medida estás de acuerdo } \\
\text { con las siguientes afirmaciones? }\end{array}$ & $\begin{array}{l}\text { Muy de } \\
\text { acuerdo }\end{array}$ & $\begin{array}{l}\text { De } \\
\text { acuerdo }\end{array}$ & Indeciso & $\begin{array}{l}\text { En } \\
\text { desacuerdo }\end{array}$ & $\begin{array}{l}\text { Muy en } \\
\text { desacuerdo }\end{array}$ \\
\hline $\begin{array}{l}\text { Considero que los hábitos de } \\
\text { estudio son fundamentales para } \\
\text { tener un buen desarrollo escolar }\end{array}$ & $53.4 \%$ & $27.3 \%$ & $10.3 \%$ & $4.7 \%$ & $4 \%$ \\
\hline $\begin{array}{l}\text { A menudo utilizo técnicas de estudio } \\
\text { que me faciliten mi aprendizaje } \\
\text { como los mapas mentales o } \\
\text { conceptuales, esquemas, fichas } \\
\text { bibliográficas entre otros }\end{array}$ & $22.9 \%$ & $43 \%$ & $22.6 \%$ & $8.4 \%$ & $2.8 \%$ \\
\hline $\begin{array}{l}\text { Considero que es importante tomar } \\
\text { apuntes propios durante o después } \\
\text { de las clases. }\end{array}$ & $47.7 \%$ & $32.7 \%$ & $12.2 \%$ & $5.6 \%$ & $1.5 \%$ \\
\hline $\begin{array}{l}\text { Siempre que tengo una duda o } \\
\text { algún aporte que hacer durante las } \\
\text { clases no dudo en expresarlo }\end{array}$ & $25.4 \%$ & $34.2 \%$ & $27 \%$ & $7.2 \%$ & $5.9 \%$ \\
\hline $\begin{array}{l}\text { Semanalmente le dedico de } 5 \text { a } 10 \\
\text { hrs de repaso a lo visto en clase }\end{array}$ & $10.6 \%$ & $27.9 \%$ & $36.4 \%$ & $16.6 \%$ & $8.1 \%$ \\
\hline $\begin{array}{l}\text { Siempre aplico mis nuevos } \\
\text { conocimientos a problemas reales }\end{array}$ & $24.5 \%$ & $45.9 \%$ & $20.1 \%$ & $6.9 \%$ & $2.5 \%$ \\
\hline $\begin{array}{l}\text { Usualmente estudio un día antes de } \\
\text { tener un examen }\end{array}$ & $27.6 \%$ & $40.5 \%$ & $20.1 \%$ & $6.9 \%$ & $5 \%$ \\
\hline $\begin{array}{l}\text { Tomo descansos durante mis horas } \\
\text { de estudio }\end{array}$ & $29.8 \%$ & $41.5 \%$ & $18.8 \%$ & $5.6 \%$ & $4 \%$ \\
\hline $\begin{array}{l}\text { Considero que el realizar un plan de } \\
\text { estudios de mis tareas semanales } \\
\text { es de ayuda para tener una mejor } \\
\text { planificación de mi tiempo }\end{array}$ & $36.1 \%$ & $39.6 \%$ & $15.7 \%$ & $5 \%$ & $3.4 \%$ \\
\hline $\begin{array}{l}\text { Siempre investigo después de } \\
\text { clases los temas vistos para reforzar } \\
\text { los conocimientos adquiridos }\end{array}$ & $18.2 \%$ & $36.4 \%$ & $30.8 \%$ & $10 \%$ & $4.4 \%$ \\
\hline mas & & & & & \\
\hline
\end{tabular}

Fuente: Elaboración propia a partir de los resultados obtenidos.

Los hábitos de estudio es uno de los mejores predictores del rendimiento académico en los estudiantes de los diferentes niveles educativos. Es por ello, que describimos algunas características de como un grupo de estudiantes de la Universidad Autónoma de León acostumbra a enfrentarse al estudio, así como a la organización de su tiempo y técnicas que utiliza para estudiar. Si bien, $80.7 \%$ de los estudiantes encuestados están de acuerdo que los hábitos de estudio son esenciales para tener un buen desempeño académico, sin embargo, sólo $65.9 \%$ señala que a menudo utiliza técnicas de estudio que me faciliten mi aprendizaje como los mapas mentales o conceptuales, esquemas, fichas bibliográficas entre otros. 
Estudiar con antelación es considerado uno de los hábitos estudios más importantes para éxito escolar, de acuerdo a la tabla $1,38.5 \%$ de los participantes están de acuerdo que semanalmente le dedican de 5 a 10 horas de repaso a lo visto en las clases, y $68.1 \%$ con frecuencia estudia un día antes de tener un examen. Aunque el hábito de estudiar con antelación no es uno de los hábitos que más practican este grupo de estudiantes, si tienen el hábito de tomar descansos durante sus horas de estudio.

La planificación del estudio es importante para mejorar el rendimiento escolar, ya que fomenta la organización del tiempo evitando la acumulación de tareas o actividades. Sobre esta línea, $75.7 \%$ de los encuestados están de acuerdo que realizar un plan de estudios de las tareas semanales es de ayuda para tener una mejor planificación del tiempo, pero debe estar acompañado de los hábitos de ser activo en clases realizando preguntas y tener una buena técnica para tomar apuntes. En este sentido, $59.6 \%$ de los estudiantes señalan que si tienen una duda o algún aporte que hacer durante las clases no dudan en expresarlo y $80.4 \%$ están de acuerdo que es importante tomar apuntes propios durante o después de las clases.

La variable de análisis que refiere al rol del estudiante se analiza desde la perspectiva de Escudero (1992), Martínez (2010) Rugeles, Mora \& Metaute (2013) quienes contemplan la capacidad de autogestión, la autodisciplina, el autoaprendizaje, el análisis crítico y reflexivo, el trabajo colaborativo y la ética profesional como las características del rol del estudiante virtual, y de los cuáles se presentan a continuación los resultados:

Tabla 2 Rol del estudiante en la modalidad virtual

\begin{tabular}{|l|l|l|l|l|l|}
\hline $\begin{array}{l}\text { En relación al rol del estudiante } \\
\text { en la modalidad virtual, ¿en qué } \\
\text { medida estás de acuerdo con las } \\
\text { siguientes afirmaciones? }\end{array}$ & $\begin{array}{l}\text { Muy de } \\
\text { acuerdo }\end{array}$ & $\begin{array}{l}\text { De } \\
\text { acuerdo }\end{array}$ & Indeciso & $\begin{array}{l}\text { En } \\
\text { desacuerdo }\end{array}$ & $\begin{array}{l}\text { Muy en } \\
\text { desacuerdo }\end{array}$ \\
\hline $\begin{array}{l}\text { Me mantengo con una actitud } \\
\text { proactiva y positiva para aprender } \\
\text { en esta nueva modalidad }\end{array}$ & $35.5 \%$ & $41.5 \%$ & $15 \%$ & $5.6 \%$ & $2.2 \%$ \\
\hline $\begin{array}{l}\text { Se me dificulta el manejo de } \\
\text { algunas aplicaciones o plataformas } \\
\text { que manejan mis profesores. }\end{array}$ & $14.1 \%$ & $29.8 \%$ & $20.4 \%$ & $18.2 \%$ & $17.3 \%$ \\
\hline $\begin{array}{l}\text { No tengo dificultades para trabajar } \\
\text { en equipo }\end{array}$ & $36.1 \%$ & $33.6 \%$ & $19.8 \%$ & $6.2 \%$ & $4 \%$ \\
\hline $\begin{array}{l}\text { Me considero una persona } \\
\text { responsable que comparte sus } \\
\text { experiencias y conocimientos. }\end{array}$ & $41.8 \%$ & $37.7 \%$ & $12.8 \%$ & $4.7 \%$ & $2.8 \%$ \\
\hline $\begin{array}{l}\text { Siempre investigo antes de las } \\
\text { clases los temas a tratar para } \\
\text { tener un conocimiento más amplio. }\end{array}$ & $9.1 \%$ & $31.7 \%$ & $38.6 \%$ & $12.5 \%$ & $7.8 \%$ \\
\hline $\begin{array}{l}\text { Tengo las habilidades de } \\
\text { comparación, análisis y síntesis. }\end{array}$ & $27.6 \%$ & $44.9 \%$ & $19.8 \%$ & $5 \%$ & $2.5 \%$ \\
\hline
\end{tabular}




\begin{tabular}{|l|l|l|l|l|l|}
\hline $\begin{array}{l}\text { Considero que he aplicado mi ética } \\
\text { profesional al realizar mis trabajos } \\
\text { y/o exámenes }\end{array}$ & $40.5 \%$ & $40.5 \%$ & $12.2 \%$ & $3.1 \%$ & $3 . \%$ \\
\hline $\begin{array}{l}\text { Me considero una persona } \\
\text { autónoma, activa y participativa } \\
\text { al momento de adquirir } \\
\text { conocimientos y habilidades para } \\
\text { mi autoformación. }\end{array}$ & $40.5 \%$ & $38.6 \%$ & $12.2 \%$ & $4.4 \%$ & $4 \%$ \\
\hline $\begin{array}{l}\text { El ser un estudiante en modalidad } \\
\text { virtual me convierte en un sujeto } \\
\text { activo dentro de mi propio } \\
\text { aprendizaje. }\end{array}$ & $31.1 \%$ & $46.2 \%$ & $15 \%$ & $4 \%$ & $3.4 \%$ \\
\hline $\begin{array}{l}\text { Considero que el ser un estudiante } \\
\text { virtual me permite desarrollar } \\
\text { la capacidad de autogestión, } \\
\text { autodisciplina y autoaprendizaje } \\
\text { para alcanza mis metas } \\
\text { profesionales. }\end{array}$ & $34.5 \%$ & $40.5 \%$ & $15 \%$ & $6.4 \%$ & $3.4 \%$ \\
\hline
\end{tabular}

Fuente: Elaboración propia a partir de los resultados obtenidos.

En modalidad virtual el estudiante se identifica como un sujeto activo, de manera que $77 \%$ de los estudiantes encuestados señalan que mantienen con una actitud proactiva y positiva para aprender en esta nueva modalidad; mientras $72.5 \%$ está de acuerdo que tienen las habilidades de comparación, análisis y síntesis. Asimismo, la autogestión es una característica indispensable en el proceso de enseñanza aprendizaje de la modalidad con uso de tecnologías, por lo que $75 \%$ señalan que ser un estudiante virtual le permite desarrollar la capacidad de autogestión, autodisciplina y autoaprendizaje para alcanzar sus metas profesionales.

Una habilidad requerida en la educación a distancia o virtual es la autonomía, ya que el estudiante debe ser responsable de sus elecciones y toma de decisiones. En este sentido, $79.1 \%$ de los estudiantes consideran ser una persona autónoma, activa y participativa al momento de adquirir conocimientos y habilidades para la autoformación. De igual forma, $81 \%$ consideran que han aplicado su ética profesional al realizar sus trabajos y/o exámenes, lo cual evidencia que se responsabilizan de sus actos. Asimismo $79.5 \%$ señalan que son personas responsables que comparten sus experiencias y conocimientos.

Por otra parte, el trabajo colaborativo es otra característica importante que se debe desarrollar el estudiante de la modalidad virtual, en este rubro $69.7 \%$ de los estudiantes encuestados están de curdo que no presentan dificultades para trabajar en equipo, sin embargo, a $43.9 \%$ de los estudiantes se le dificulta el manejo de algunas aplicaciones o plataformas que manejan los profesores.

De igual forma, en apego a los planteamientos hechos por Brooks (2020), existe un impacto psicológico generado por la situación actual de confinamiento por pandemia, que se manifiesta a través de sentimientos de frustración y aburrimiento, negatividad y apatía, emociones de las cuáles se presentan los siguientes resultados: 
Tabla 3 Situación emocional y de estrés por modalidad virtual

\begin{tabular}{|c|c|c|c|c|c|}
\hline $\begin{array}{l}\text { En relación a la situación } \\
\text { emocional y de estrés por } \\
\text { modalidad virtual, ¿en qué } \\
\text { medida estás de acuerdo con las } \\
\text { siguientes afirmaciones? }\end{array}$ & $\begin{array}{l}\text { Muy de } \\
\text { acuerdo }\end{array}$ & $\begin{array}{l}\text { De } \\
\text { acuerdo }\end{array}$ & Indeciso & $\begin{array}{l}\text { En } \\
\text { desacuerdo }\end{array}$ & $\begin{array}{l}\text { Muy en } \\
\text { desacuerdo }\end{array}$ \\
\hline $\begin{array}{l}\text { Mis emociones influyen en esta } \\
\text { nueva modalidad }\end{array}$ & $48.4 \%$ & $29.8 \%$ & $13.2 \%$ & $5.9 \%$ & $2.5 \%$ \\
\hline $\begin{array}{l}\text { Las emociones que he } \\
\text { experimentado durante este } \\
\text { periodo han sido positivas } \\
\text { (alegría, entusiasmo, satisfacción, } \\
\text { confianza, seguridad, etc.) }\end{array}$ & $16.6 \%$ & $33.9 \%$ & $33.6 \%$ & $11 \%$ & $4.7 \%$ \\
\hline $\begin{array}{l}\text { Las emociones que he } \\
\text { experimentado durante este } \\
\text { periodo han sido negativas } \\
\text { (frustración, aburrimiento, tristeza, } \\
\text { fastidio, enfado, desesperación, } \\
\text { estrés, etc.). }\end{array}$ & $24.5 \%$ & $34.5 \%$ & $27 \%$ & $6.9 \%$ & $6.9 \%$ \\
\hline $\begin{array}{l}\text { Esta nueva modalidad me ha } \\
\text { hecho pensar en abandonar tus } \\
\text { estudios. }\end{array}$ & $13.8 \%$ & $15 \%$ & $17.9 \%$ & $17.9 \%$ & $35.2 \%$ \\
\hline $\begin{array}{l}\text { Tengo un buen ambiente de } \\
\text { aprendizaje en tus clases }\end{array}$ & $19.1 \%$ & $45.5 \%$ & $27 \%$ & $5 \%$ & $2.8 \%$ \\
\hline $\begin{array}{l}\text { En algún momento me sentí } \\
\text { rechazado e ignorado }\end{array}$ & $16.0 \%$ & $20.4 \%$ & $26.1 \%$ & $17.6 \%$ & $19.4 \%$ \\
\hline $\begin{array}{l}\text { Consideras que el apoyo que te } \\
\text { dan tus profesores es un aspecto } \\
\text { importante para tu motivación }\end{array}$ & $26.1 \%$ & $38.6 \%$ & $25.1 \%$ & $5.9 \%$ & $4 \%$ \\
\hline $\begin{array}{l}\text { Consideras que la forma en la } \\
\text { que los profesores te hacen llegar } \\
\text { nuevos conocimientos influye en tu } \\
\text { motivación escolar }\end{array}$ & $30.8 \%$ & $43.0 \%$ & $19.4 \%$ & $3.1 \%$ & $3.4 \%$ \\
\hline $\begin{array}{l}\text { Con frecuencia siento cansancio } \\
\text { durante tus clases virtuales }\end{array}$ & $29.2 \%$ & $29.5 \%$ & $22.9 \%$ & $10.3 \%$ & $5.9 \%$ \\
\hline $\begin{array}{l}\text { Tengo buena comunicación con } \\
\text { mis compañeros. }\end{array}$ & $22.6 \%$ & $38.9 \%$ & $23.5 \%$ & $9.4 \%$ & $4.4 \%$ \\
\hline
\end{tabular}

Fuente: Elaboración propia a partir de los resultados obtenidos. 
Si bien, en la educación presencial como en la virtual el actor principal es el estudiante, es por ello, que el docente o facilitador debe generar un ambiente de aprendizaje que permita la interacción y participación de este. Sin embargo, el $64.6 \%$ de los estudiantes encuestados están de acuerdo que tienen un buen ambiente de aprendizaje en sus clases; mientras un $73.8 \%$ consideran que la forma en que los profesores hacen llegar los nuevos conocimientos influye en su motivación escolar, y este aspecto de apoyo por parte del facilitador, $64.7 \%$ de los estudiantes están de acuerdo que el apoyo que dan los profesores es un aspecto importante para tu motivación.

La motivación en el proceso de aprendizaje es esencial para un buen desempeño y la situación emocional del estudiante, sobre esta línea de las emociones que experimentan los estudiantes en la modalidad se puede visualizar que el $50.5 \%$ de los estudiantes encuestados han experimentado emociones positivas (alegría, entusiasmo, satisfacción, confianza, seguridad, etc.); mientras el $59 \%$ están de acuerdo que está modalidad han emociones negativas como frustración, aburrimiento, tristeza, fastidio, enfado, desesperación, estrés, etc.. En consecuencia, $78.2 \%$ de los estudiantes están de acuerdo que sus emociones influyen en su proceso de aprendizaje en la nueva modalidad de aprendizaje.

A pesar de que en la nueva modalidad los estudiantes experimentan emociones negativas, sólo $28.8 \%$ de los estudiantes han pensado en algún momento abandonar tus estudios y $36.4 \%$ aceptan que se han sentido rechazos durante el proceso de enseñanza de aprendizaje. Además $58.6 \%$ señala que con frecuencia sienten cansancio durante tus clases virtuales.

\section{CONCLUSIÓN}

El instrumento, aplicado en su primera versión, ha permitido identificar tres variables que se consideran de relevancia para el éxito escolar en un ambiente universitario.

De acuerdo a los planteamientos que realizan los autores Escudero (1992), Martínez (2010) Rugeles, Mora \& Metaute (2013), en los que se identifica la autogestión, la autodisciplina, el autoaprendizaje, análisis crítico y reflexivo, el trabajo colaborativo ética profesional como elementos influyentes en el rol de estudiante en modalidad a distancia; un alto porcentaje de la muestra como un estudiante consciente del nivel de auto regulación que implica la modalidad virtual, una figura pro-activa $y$ positiva en su rol de aprendiz de la misma forma facilita la información de la forma en la que se asumen como profesionales competente, auto gestionado, auto disciplinado y con sentido ético; asume su rol responsable y autónomo dentro de su propio proceso de aprendizaje.

Otra información que nos permite obtener el instrumento en esta fase exploratoria, son las dificultades que los estudiantes enfrentan en relación al manejo de las plataformas de aprendizaje utilizadas ante la nueva normalidad, para la continuidad de la formación profesional. En este sentido, se considera que dentro de su rol se asume la importancia de poseer los recursos y establecer las condiciones necesarias para su óptimo desempeño.

Por su parte Cartagena (2008), expresa la importancia de los hábitos de estudio para lograr un proceso de comprensión de contenidos complejos, generar estadios de concentración en los estudiantes, lo que lleva a la optimización de su esfuerzo para aprender; el instrumento permite identificar la percepción que los estudiantes tienen sobre la importancia de los hábitos, de igual forma genera información sobre las técnicas de estudio utilizadas para facilitar 
sus procesos cognitivos como por ejemplo fichas bibliográficas mapas mentales o conceptuales, esquemas, entre otros. Las horas de dedicación al estudio es otra información que el instrumento nos permite obtener, siendo más del $50 \%$ de los estudiantes quienes destinan sólo 24 horas antes de la aplicación de un examen, tiempo para el estudio o repaso de los temas sobre los que se van a examinar.

Los hábitos de estudio son importantes para el desarrollo de los estudios universitarios y a pesar de que cuentan con información sobre los beneficios de los mismos, existe un número reducido que los implementan de forma adecuada, la gran mayoría sólo recurre a descansos entre actividades, no todos toman apuntes entre otras características de relevancia.

Sobre la variable estrés como consecuencia de la formación profesional en modalidad virtual, Brooks (2020), plantea la existencia de un impacto psicológico como consecuencia ineludible de la situación actual de confinamiento por pandemia caracterizando algunos sentimientos como respuesta a ello como: frustración y aburrimiento, negatividad y apatía. El instrumento permite obtener información sobre la motivación que el ambiente de aprendizaje diseñado por el profesor genera en su estado emocional. Estableciendo que la figura del docente como facilitador del proceso es un elemento determinante en las emociones que se gestan en el estudiante, y que llegan a ser consecuentes con su permanencia o deserción escolar.

Información relevante generada por el instrumento, es la identificación de más del $50 \%$ de la población encuestada que se ha experimentado emociones negativas, es decir que la modalidad virtual a consecuencia de la pandemia por COVID-19, les ha generado sentimientos de estrés, tristeza, enfado, fastidio, aburrimiento y frustración, lo cual está afectando su salud y obstaculizando su proceso de aprendizaje.

Finalmente, se expresa que el instrumento en esta fase de prueba genera información de relevancia para conocer la percepción de los estudiantes universitarios en relación a su rol como estudiante en modalidad virtual, sus hábitos de estudio y su situación emocional. Se plantea una segunda aplicación modificando el instrumento de forma tal que pueda tener un mayor alcance y permitiendo se le realicen pruebas estadísticas para su validación.

\section{REFERENCIAS BIBLIOGRÁFICAS}

Área, M. y Adell, J. (2009): e-Learning: Enseñar y aprender en espacios virtuales. Tecnología Educativa. La formación del profesorado en la era de Internet. Aljibe, Málaga, págs. 391-424.Recuperado de: https://www.researchgate.net/ publication/216393113_E_Learning_ ensenar_y_aprender_en_espacios_ virtuales

Brooks, S, Webster, K., Smith, L, Woodland, L, Wessely, S, Greenberg, N, y Rubin, G. J. (2020). The psychological impact of quarantine and how to reduce it: Rapid review of the evidence. The Lancet, 395(10227), 912-920. doi:10.1016/ S0140-6736(20)30460-8

Cartagena, M. (2008). "Relación entre la autoeficacia, el rendimiento escolar y los hábitos de estudio de secundaria", Revista Iberoamericana sobre Calidad, Eficacia y cambio en Educación. Recuperado de: http://www.rinace.net/ arts/vol6num3/art3.pdf 
Chehaibar, L. (2020). Flexibilidad curricular. Tensiones en tiempos de pandemia. Educación y pandemia. Una visión académica, 83-91.

Escudero, Juan. (1992). La integración escolar de las nuevas tecnologías de la información. Infodidac, Revista de Informática y Didáctica.

Martínez F, Agustín J, Moreno J, (2010). Análisis delapercepción ética entre profesionales del sector educativo. Revista de Investigación Educativa, 28 (2), 385401. Recuperado de: https://www. redalyc.org/pdf/2833/283321930010. pdf

INEGI (2018). Encuesta Nacional sobre la Disponibilidad y uso de TIC en hogares. México: INEGI. Recuperado de https:// www.inegi.org.mx/temas/ticshogares/

Instituto Internacional para la Educación Superior en América Latina y el Caribe. (2020). COVID-19 y educación superior: De los efectos inmediatos al día después. Organización de las Naciones Unidas para la Educación, la Ciencia y la Cultura. http://www.ie-salc.unesco.org/ wp-content/uploads/2020/05/COVID19-ES-130520.pdf

Maldonado, G., de los Ángeles, M., Stratta, A., Barrera, A. y Zingaretti, L. (2020). La educación superior en tiempos de covid-19. Análisis comparativo México Argentina. Revista de Investigación en Gestión Industrial, Ambiental, Seguridad y Salud en el Trabajo, 3560.

Morelos, E., de Morelos, A. D. E., \& de Nayarit, A. (2020). Impacto psicológico en estudiantes universitarios mexicanos por confinamiento durante la pandemia por Covid-19.
PNUD (2019), "Panorama general. Informe sobre Desarrollo Humano 2019. Más allá del ingreso, más allá de los promedios, más allá del presente: Desigualdades del desarrollo humano en el siglo xxi", Nueva York, consultado el 20 de marzo, 2020.

Rugeles P., Mora B., Metaute P., (2015). El rol del estudiante en los ambientes educativos mediados por las TIC. Rev. Lasallista, vol.12 no.2. Recuperado de:

ht t p://www.scielo.org.co/scielo. php?script=sci_arttext\&pid=S179444492015000200014\#: :text=Algunas $\% 20$ caracter\%C3\%ADsticas\%20del\%20rol\%20 del,colaborativo $\% 2 \mathrm{C} \% 20$ fundamental $\% 20$ para\%20contribuir\%20al

Saini, V., \& Mitteer, D. R. (2020). A review of investigations of operant renewal with human participants: Implications for theory and practice. Journal of the Experimental Analysis of Behavior. doi: 10.1002/jeab.562

Salazar, M. (2020). Escenarios de conversación y aprendizaje. Cultura educación y sociedad, 11(1), 219-234. ht-tps://doi. org/10.17981/cultedusoc.11.1.2020.16

Sanz, I, Sainz, J., \& Capilla, A. (2020). Efectos de la crisis del coronavirus en la educación. Organización de Estados Iberoamericanos para la Educación, la Ciencia y la Cultura (OEI).

Sousa Santos, Boaventura de (2020), La cruel pedagogía del virus, Buenos Aires, clacso, consultado el 23 de octubre de 2020. 
UNESCO IESALC. (2020). Covid-19 y educación superior: de los efectos inmediatos al día después. Análisis de impactos, respuesta y recomendaciones. Recuperado de

http://www.iesalc.unesco.org/wp-content/ uploads/2020/04/COVID-19-060420ES-2.pdf

World Economic Forum (WEF). (2020). 3 ways the coronavirus pandemic could reshape education. Recuperado de https://www. weforum.org/agenda/2020/03/3-wayscoronavirus-is-reshaping-educationand-what-changes-might-be-here-tostay/ 